\title{
Plasma Flow Past Cometary and Planetary Satellite Atmospheres
}

\author{
Michael R. Combi and Tamas I. Gombosi \\ The University of Michigan, Ann Arbor, Michigan \\ Konstantin Kabin \\ The University of Alberta, Edmonton, Alberta, Canada
}

The tenuous atmospheres and ionospheres of comets and outer planet satellites share many common properties and features. Such similarities include a strong interaction with their outer radiation, fields and particles environs. For comets the interaction is with the magnetized solar wind plasma, whereas for satellites the interaction is with the strongly magnetized and corotating planetary magnetospheric plasma. For this reason there are many common or analogous physical regimes, and many of the same modeling techniques are used to interpret remote sensing and in situ measurements in order to study the important underlying physical phenomena responsible for their appearances. We present here a review of various modeling approaches which are used to elucidate the basic properties and processes shaping the energetics and dynamics of these systems which are similar in may respects.

\section{INTRODUCTION.}

Comets and several planetary satellites possess thin tenuous atmospheres which interact with their outer magnetized plasma environs and solar radiation to ultimately drive the atmospheres into escape and producing ions which mass-load the outer plasma. In the case of comets the gravity is vanishingly small $\left(\sim 10^{-4}\right.$ times gravity at the Earth's surface). In the case of the planetary satellites (e.g., Jupiter's largest four moons) gravity is small, but the plasma impact processes are strong enough that only a tenuous atmosphere is maintained (see Chapter III.3) and a large neutral escape rate, comparable to a moderate comet, is maintained. For these reasons similar approaches are used for modeling various observed phenomena in order to study the underlying causes. The innermost neutral atmospheres have been treated with hydrodynamic approaches, and the outermost atmospheres have been treated with the assumption of vacuum free-flow. In between these two regions is a complicated kinetic region where collision mean free paths are large compared with relevant spatial scales, but not large enough to ignore. Interactions with cooling infrared radiation also become non-LTE because of decoupling between rotational and kinetic temperatures.

Magnetohydrodynamic (MHD) approaches have been used to study the large scale interactions of impacting magnetized plasma with the ionospheres of these bodies. For comets, it is the solar wind with its frozen-in magnetic field which impacts the cometary ionosphere. For planetary satellites, it is the corotating (or nearly corotating) plasma flow frozen into the planet's strong dipolar magnetic field which impacts the satellite ionosphere. Mass-loading via photo- and charge impact ionization and charge exchange are important for explaining the overall structure of both kinds of interactions.
For comets, this interaction produces the often spectacular ion tail, which is millions of kilometers long. For planetary satellites, the Galileo spacecraft and ground-based and Earthorbit based observing have revealed an array of auroral type emissions.

\section{SOLAR WIND INTERACTION WITH COMET ATMOSPHERES AND IONOSPHERES}

\subsection{The Cometary Atmosphere}

A well developed cometary atmosphere extends to distances several orders of magnitude larger than the size of the nucleus. Self-consistent models of cometary atmospheres must describe phenomena taking place at several spatial and temporal scales ranging from the initiation of the transonic dusty gas flow near the nucleus (with typical scalelength of $10^{-2}-10^{-1} \mathrm{~km}$ ) to fast chemical reactions (typical scales are $10^{\circ}$ $10^{2} \mathrm{~km}$ ) to the formation of the cometary ionosphere (typical scales are $10^{3}-10^{4} \mathrm{~km}$ ), to the solar wind interaction of the cometary atmosphere (typical scales are $10^{5}-10^{7} \mathrm{~km}$ )..

Today little direct observational data are available on the near surface gas, dust and plasma environments of cometary nuclei. Our present understanding of this region is based on dust coma images from the Giotto spacecraft Keller [1986] and dusty-gasdynamic calculations having varying levels of sophistication [Crifo el al., 1999a]. Verification is primarily based on comparing model predictions hundreds of nucleus radii from the nucleus with in situ and remote observations.

The bulk velocity of the outflowing gas is slightly subsonic at the surface (the effusion would be sonic without any gas-dust interaction: the momentum loss to the entrained dust particles results in a slightly subsonic outflow). Frequent gas-dust collisions erode and drag away grains from 
the surface and accelerate them to significant velocities within a few nucleus radii. Small dust particles $(<10 \mu \mathrm{m})$ can be fully accommodated to the gas flow and reach velocities up to several hundreds of $\mathrm{m} / \mathrm{s}$, while larger grains attain much slower velocities (the dust terminal velocity is proportional to the inverse of the grain size) [Gombosi et al., 1986]. The adiabatically expanding gas converts most of its internal energy to bulk motion, while it loses some of its momentum and energy to the dust flow. This process is expected to result in a very cold (several tens of $\mathrm{K}$ ) hypersonic gas flow in the immediate vicinity of the nucleus. The problem is also complicated by the fact that dust particles, especially the more volatile CHON particle can serve as sources for gaseous species. These particles get their name because they are composed of mostly compounds from carbon, hydrogen, oxygen and nitrogen, as opposed to the more refractory silicate dust composed of silicon and metal oxide compounds. CHON particles can thus more easily fragment and vaporize, modifying the gas and dust mass, momentum and energy densities.

Most of the species observed in cometary atmospheres are chemically unstable radicals, which clearly are photodestruction products of more stable parent molecules. Chemical reactions between evaporated parent molecules are very slow and of secondary importance, while dissociation and ionization by solar UV radiation produce highly reactive radicals and ions.

Most neutral coma modeling deals with the two limiting conditions which occur far from and near the nucleus: freemolecular and fluid-flow models. Free-molecular models describe the distributions of molecules in the outer coma where intermolecular collisions are rare. These include radial outflow models [Haser 1957; Haser 1966; Wallace and Miller, 1958; Keller and Meier, 1976], and non-radial models accounting for isotropic ejection of daughter species [Combi and Delsemme, 1980a; Festou 1981]. Fluid models describe the bulk properties of various fluids (gases, dust, and plasma) that are collisionally coupled in the inner coma. Examples of these are gas-phase chemistry models [Giguere and Huebner, 1978; Mitchell et al., 1981; Cochran 1985; Allen et al., 1987], combined chemical-dynamic models [Huebner and Keady, 1983], dusty-gasdynamic models [Marconi and Mendis, 1983, Gombosi et al. 1986], and multidimensional dusty-gasdynamic models [Kitamura 1986; Kömle and Ip. 1987; Körösmezey and Gombosi, 1990; Crifo 1995; Crifo et al., 1999a; Crifo et al., 1999b; Combi et al., 1999].

Monte Carlo methods have bridged the gap between the fluid and free-flow regimes in the important transition region where many observations occur. After the first Monte Carlo coma model [Combi and Delsemme, 1980a], collisions were introduced [Kitamura et al., 1985] to model the spatial distribution of the $\mathrm{H}$ coma and the partial collisional thermalization of $\mathbf{H}$ atoms. Since then this approach has been significantly generalized and extended, making a selfconsistent picture of the inner and outer coma [Combi 1987; Bockelée-Morvan and Crovisier, 1987; Combi and Smyth, 1988a and 1988b; Ip 1989], added dusty-gas hydrodynamics to the inner coma [Combi 1989], a treatment for heavy species [Combi et al., 1993], and Ly- $\alpha$ radiative transfer [Combi and Feldman, 1992]. These models have been used to analyze various observations of water dissociation products and trace species in P/Halley including spatial profiles, column abundances (production rates), and Doppler line profiles. These hybrid models have even been extended to treat the large collision region of comet Hale-Bopp (1995 01). Figure 1 shows outflow velocities and kineitc temperatures at a function of distance from the nucleus for a number of heliocentric distances as calculated using a hybrid 1D-spherical hydrodynamics/Monte Carlo calculation [Combi et al., 1999]. These predicted outflow velocities and kinetic temperatures were shown to provide a reasonable explanation for radio observations of the heliocentric distance dependence of both outflow velocity and kinetic temperature in the inner

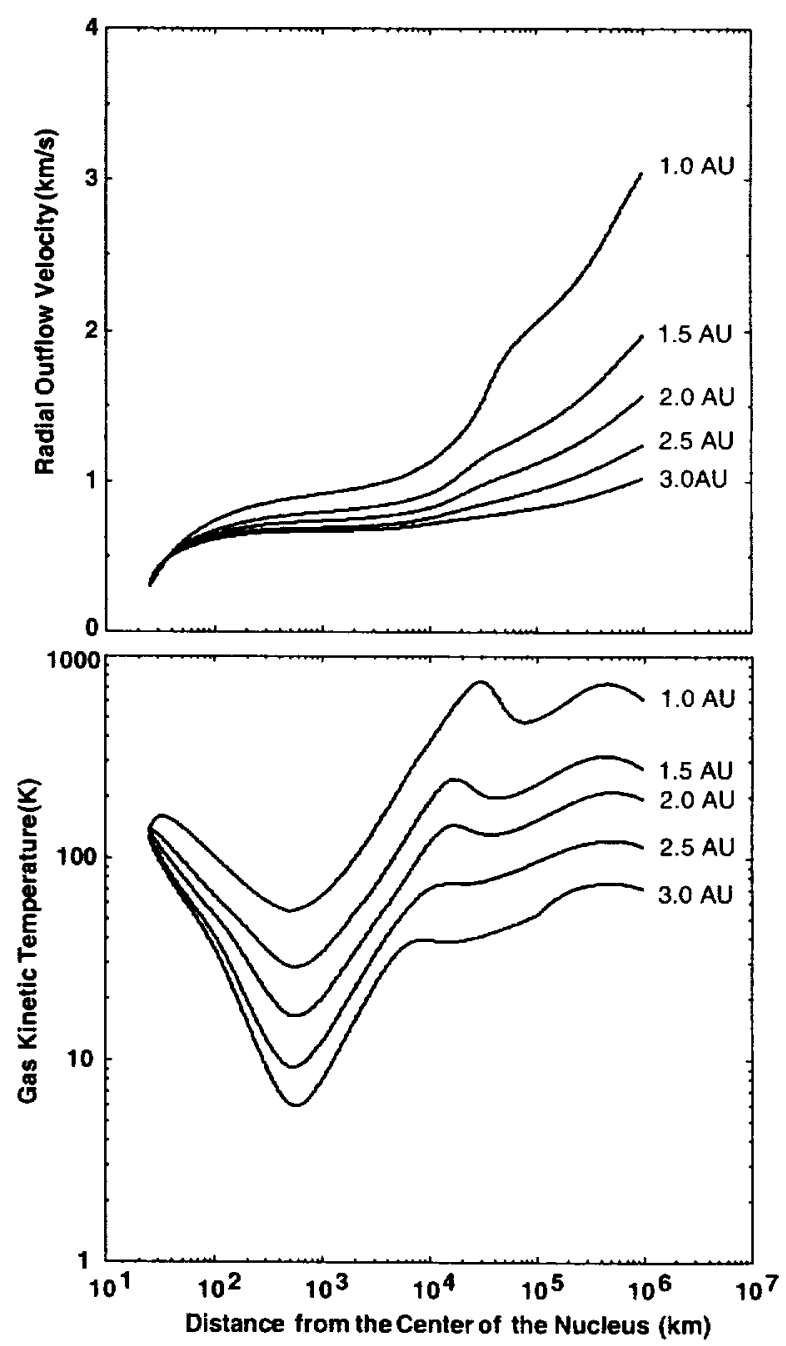

Figure 1. Radial Outflow Velocity and Kinetic Temperature in the Coma of Hale-Bopp. Shown are the results of a onedimensional spherical hybrid kinetic/dusty-gas hydrodynamic calculations from Figure 1 in the paper by [Combi et al., 1999]. Above is the variation of the radial outflow velocity with distance from the nucleus for different heliocentric distances of Comet Hale-Bopp. Below are similar curves for the gas kinetic temperature. 
coma [Biver et al., 1999] and outflow velocity in the outer coma [Colom et al., 1999].

Fully kinetic iterative test particle (ITP) models developed for describing atomic species in planetary exospheres have been applied to comets [Hodges 1990]. Xie and Mumma [1996a and 1996b] have presented improved ID spherical and a 2D axisymmetric versions. In an ITP model the trajectories and collisions of molecules of each species are computed through a background gas of the modeled species themselves, as given by their phase space velocity distribution functions. It arrives at steady-state solutions for the species distribution functions by iteratively updating the background distributions with those accumulated from the test particle trajectories.

In recent years these test particle models have been superceded by Direct Simulation Monte Carlo calculations (DSMC) orignally pioneered by Bird for high Knudsen number gas dynamics flow problems [Bird 1976 and1994]. DSMC is a direct simulation of the generalized rarefied problem which is most fundamentally described by a solution to the collisional Boltzmann equation. The combination of velocity moments of the Boltzmann equation with closure arguments and the ideal gas law can be manipulated to yield the more familiar (and tractible) equations of hydrodynamics: both the Euler and NavierStokes versions. DSMC-type methods have been applied to ionospheric and magnetospheric plasma simulations [Wilson et al., 1992; Miller and Combi, 1994, Miller et al., 1995] and earth's corona [Shematovich et al., 1994]. Pospieszalska and Johnson, 1992 and 1995] have presented 1D spherical particle simulations for Io's $\mathrm{SO}_{2}$ atmosphere using a method which is philosophically between the ITP and DSMC methods, mixing elements of both. Most recent DSMC calculations for lo's tenuous atmosphere have been published by Marconi et al. [1996] and Austin and Goldstein [2000], as we will discuss in a later section. However, of particular relevance here is that DSMC has been applied to study high Knudsen number flows in neutral cometary atmospheres [Combi 1996; Harris et al., 1997; Skorov and Rickman 1998].

Unlike the ion chemistry in the coma which is sparked by photochemistry (but where the detailed composition is controlled by fast ion-neutral reactions), the neutral chemistry is controlled in large measure by photochemistry. The composition of a "typical" comet is about $80 \%$ water, about $5 \%$ each of $\mathrm{CH}_{3} \mathrm{OH}$, and other organics, $3 \% \mathrm{CO}_{2}, 0.5 \% \mathrm{NH}_{3}$ and $<1 \% \mathrm{HCN}, \mathrm{CH}_{3} \mathrm{CN}, \mathrm{C}_{2} \mathrm{H}_{2}$, and $\mathrm{C}_{2} \mathrm{H}_{6}$ [cf. Crovisier 1999]. In addition, there is significant $\mathrm{CO}$ and $\mathrm{H}_{2} \mathrm{CO}$ in comets (up to $10-20 \%$ total $\mathrm{CO}$ in some comets), but the fraction that comes directly as a gas from the nucleus, e.g., frozen $\mathrm{H}_{2} \mathrm{CO}$, as opposed to being vaporized from an extended source of grains is uncertain. The balance is also uncertain between parent molecule gases and vaporizing grains as sources for the carbon containing radicals, $C_{2}, C_{3}$ and $\mathrm{CN}$. A fraction of the source of $\mathrm{CN}$ and $\mathrm{C}_{2}$ may be associated with grains but clearly some of the $\mathrm{CN}$ and $\mathrm{C}_{2}$ comes from photodissociation of $\mathrm{HCN}$ and $\mathrm{C}_{2} \mathrm{H}_{2}$, respectively, which are both among the parent gases emitted from the nucleus [cf. Brooke et al., 1996; Combi and Fink, 1997; Rauer et al., 1997].

\subsection{The Cometary Ionosphere}

The main photoionization product in the cometary coma is $\mathrm{H}_{2} \mathrm{O}^{+}$. These water ions transfer a proton to other neutral molecules, which have a higher proton affinity than the $\mathrm{OH}$ radical (the prime example is $\mathrm{H}_{2} \mathrm{O}^{+}+\mathrm{H}_{2} \mathrm{O}->\mathrm{H}_{3} \mathrm{O}^{+}+\mathrm{OH}$ ). The proton affinity of the parent molecules determines the reaction rate, therefore protonated ions of molecules with high proton affinity are more abundant (relative to other ions) than their parent molecules are (relative to other neutrals) [Häberli et al., 1997a]. The important species and reactions for ion chemistry in a water-dominated coma are summarized in tables by Häberli et al. [1997a].

Neutral atoms and molecules of cometary origin move along ballistic trajectories and become ionized with a characteristic ionization lifetime of $10^{5}-10^{7} \mathrm{~s}$. Freshly born ions are accelerated by the motional electric field of the highspeed solar wind flow. The ion trajectory is cycloidal, resulting from the superposition of gyration and $\mathbf{E} \times \mathbf{B}$ drift. The corresponding velocity-space distribution is a ring-beam distribution, which has large velocity space gradients and it is unstable to the generation of low frequency transverse waves. The combination of ambient and self-generated magnetic field turbulence pitch-angle scatters the newborn ions from the pickup ring. As a result of this process the pitch angles of the pickup-ring particles are scattered on the spherical velocity space shell around the local solar wind velocity [Neugebauer et al., 1991a; Motchmann et al., 1993; Coates et al., 1993].

Conservation of momentum and energy require that the solar wind be decelerated as newly born charged particles are "picked up" by the plasma flow. Continuous deceleration of the solar wind flow by mass loading is possible only up to a certain point at which the mean molecular weight of the plasma particles reaches a critical value. At this point a weak shock forms and impulsively decelerates the flow to subsonic velocities. The cometosheath is located between the cometary shock and the magnetic field free region in the innermost coma. The plasma population in the cometosheath is a changing mixture of ambient solar wind and particles picked up upstream and downstream of the shock. Near the nucleus ion-neutral chemistry and recombination starts to become more and more important. In the inner coma, an "ionopause" or "diamagnetic cavity boundary," separates the solar wind controlled magnetized plasma from the magnetic field free cometary ionosphere, the diamagnetic cavity. Deep inside the diamagnetic cavity the cometary plasma and the neutral gas are very strongly coupled by ion-neutral collisions, and they move radially outward with the same expansion velocity. See Figure 2.

\section{SATELLITE ATMOSPHERES AND IONOSPHERES WITHIN PLANETARY MAGNETOSPHERES}

The nature of the atmospheres and ionospheres of Jupiter's natural satellites lo and Europa have been treated in chapters III. 2 and III.3. Their interactions with their surrounding radiation, and particles and fields environments are very active fields of study. Various frameworks, depending on different regime-dependent approaches have been adopted in recent years, with the hope of understanding the basic global 


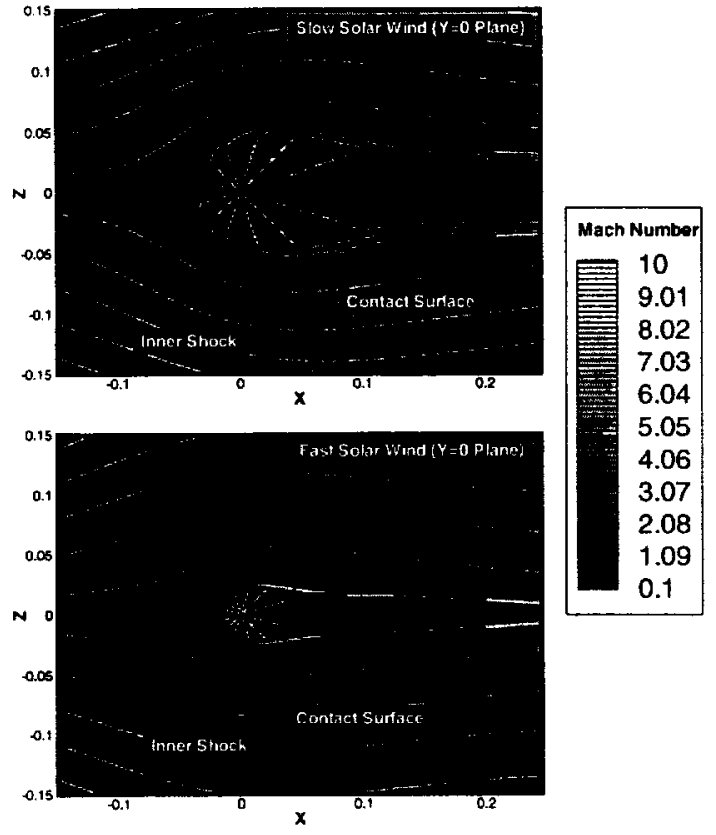

Figure 2. Comet Ionosphere Interaction with the Solar Wind. Shown is a plot of plasma flow streamlines (white) superimposed on a color plot of Mach number. The boundaries of the contact surface and diamagnetic cavity are indicated.

structure of the atmospheres, and their interactions with solar radiation and with the Jovian plasma torus environment.

\subsection{Europa's Interaction with Jupiter's Magnetosphere}

Recent theoretical work on the atmosphere/ionosphere of Europa has been done using diffusive photochemical and energy-balance models. Being constrained by the observations of Hall et al. [1995] and Brown [1996], Ip [1996] have made 1D-spherical calculations that predict a lower atmosphere with a moderate exponential scale height and an extended corona. Saur et al. [1998] performed a study of 3D aspects of the interaction of Jupiter's plasma torus with Europa's atmopshere. They adopted multiple 1D hydrostatic-type diffusive profiles for the atmosphere and account for chemistry and energy balance and the impacting plasma torus. They solved for the effects of currents and electric fields assuming a constant and uniform magnetic field. The recent flybys of Europa by the Galileo orbiter indicate the presence of an ionosphere [Kliore et al., 1997], roughly consistent with expectations from the $O$ detection, and a disturbed local magnetic field [Kivelson et al., 1997; Neubauer 1998] which could be accounted for by an intrinsic field or an induced field.

Figures 3 and 4 show the main results of the simulations of Galileo's E4 Europa flyby by Kabin et al. [1999b]. It was found that the best agreement with observations of Kivelson et al. [1997] and Gurnett et al. [1998] can be obtained by assuming a total mass loading rate of $7 \times 10^{25} \mathrm{O}_{2}{ }^{+}$ions per second [Kliore et al,. 1997]. The best agreement with observations was obtained when mass-loading was constrained to the ram side, assuming that the dominant ionization mechanism is electron impact [Kliore et al., 1997]. Mass loading alone was able to account for the observed signature in $B_{z}$, while reproducing the $B_{x}$ and $B_{y}$ signatures required the inclusion of an intrinsic magnetic dipole of $65 \mathrm{nT}$ along the $\mathrm{y}$ axis. This intrinsic magnetic field is consistent with the presence of a subsurface ocean [Khurana et al., 1998; Neubauer 1999; Zimmer et al., 2000] which would produce an induced intrinsic field in the $(x, y)$ plane with $\mathrm{B}_{\mathrm{y}}$ being the major component [Khurana et al., 1998]. However, the induced magnetic field deduced by [Khurana et al., 1998] is somewhat larger than this "best fit". This work required adopting a $20^{\circ}$ rotation of the upstream torus flow toward the direction of Jupiter. A recent paper by Paranicas et al. [2000] suggests that this might not be a property of the actualy bulk flow of the torus ions but simply a local effect such as that which results from the electric field rotation in electrodynamic simulations [Saur et al., 1998].

A later two-species MHD calculation for the plasma interaction near Europa has most recently been done by Liu et al. [2000]. In this calculation the impacting plasma torus ions (dominated by $\mathrm{O}^{++}, \mathrm{S}^{++}$, and $\mathrm{S}^{+++}$) represent one species and the local pickup ions (dominated by $\mathrm{O}_{2}{ }^{+}$) are the other. The model parameters and form were otherwise similar to those of [Kabin et al., 1999b]. One difference is that with the choice of two species there was freedom to be able to better estimate electron density because of the different mean charge states of the ambient torus ions $(=2)$ and the local pickup ions $(=1)$. Furthermore, the results of the Galileo Plasma Science instrument [Paterson et al., 1999] appeared in the literature in the intervening time. Figures $5-7$ show the results of the comparison of various Galileo particles and fields instrument measurements during the E4 flyby with the two-species MHD calculations of Liu et al. [2000].

The agreement with the measured electron density data from the plasma wave instrument is quite good. Two density peaks in the wake region correspond to the closest approach and the center of the true corotation wake. These peaks consist mainly of $\mathrm{O}_{2}^{+}$. The ambient plasma torus ions actually show a small minimum density inside the wake, consistent with calculations of flow past an obstacle when there is no mass-loading. See, for example, the first reference lo model by Combi et al. [1998a], who considered a perfectly conducting sphere and no mass-loading. An essentially empty wake resulted. The ion speed in Figure 6 shows approximately the correct qualitative behavior; however, the model produces more of a velocity decrease in the wake. Similarly the temperature shows the right qualitative structure; however, the model considers total plasma temperature which effectively has contributions from both ion populations and electrons, whereas the observations are only of the ions. In this regime of the plasma torus the total electron temperature is uncertain, but it is believed that there is an important contribution from a population of high energy electrons which is non-negligible toward the total plasma pressure. For this reason the plot also compares twice the measured ion temperature with the MHD plasma 

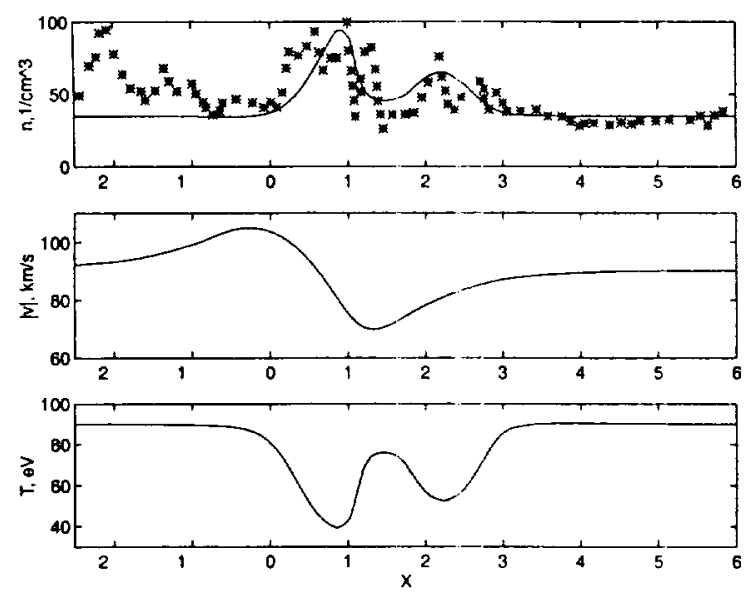

Figure 3. Comparison of measured and simulated densities, as well as simulated speed and temperature profiles along the Galileo E4 flyby irajectory.
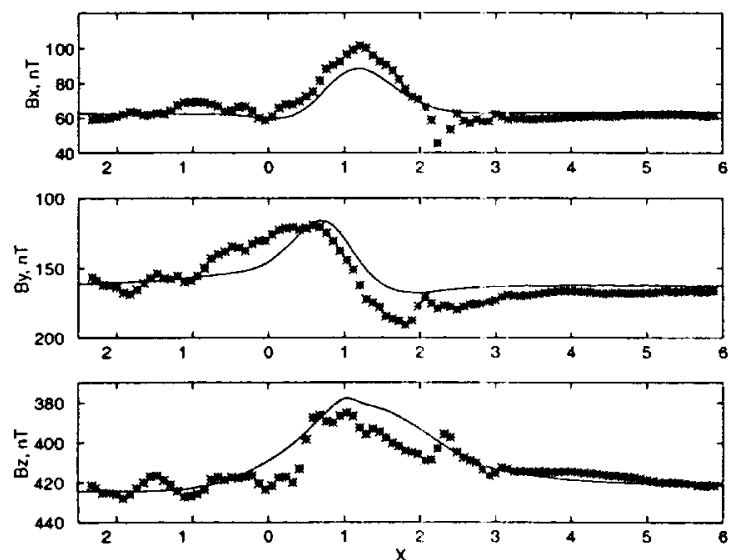

Figure 4. Comparison of measured and simulated magnetic field vectors (solid lines) along the Galileo E4 flyby trajectory.

temperature. Again the structure with three maxima is qualitatively reproduced.

Lastly, the comparison with the Galileo magnetometer measurements in Figure 7 is also quite good. The approach of Kabin et al. [1999b] and Liu et al. [2000] has been to leave the magnitude and orientation of the internal Europa magnetic field as a free parameter and use the comparison with the data to converge to the best "fit," rather than assuming from the beginning that an internal induced field from a sub-surface ocean was responsible. The magnitude and direction of the magnetic field in both of these studies, is in fact consistent with this picture of the induced internal field picture suggested by Khurana et al. [1998] and Neubauer [1999], providing an independent confirmation.

\subsection{Io's Interaction with Jupiter's Magnetosphere}

Our understanding of the interaction of Io with Jupiter's corotating plasma goes back to the discovery of Io-related decametric radio emission discovered by [Bigg 1964] and the unipole inductor model which was introduced to explain it [Goldreich and Linden-Bell, 1969]. The basic premise of the unipole inductor picture has recently been questioned by Russell and Huddleson, [2000]. There were a number of theoretical studies of this interaction during the immediate post-Voyager era (sees [Hill 1983] for a post-Voyager review). Early theoretical work was often done either in the context of a "thin" atmosphere (e.g., see Cloutier [1978]) indicative of the surface temperature $(\sim 130 \mathrm{~K})$, or "thick" extended neutral atmosphere (e.g. see Goertz [1980]) more indicative of volcanic temperatures $(-1000 \mathrm{~K})$. Subsequent evidence (see the review by Lellouch [1996]) seems to indicate a mixed picture of the global atmosphere, which has a large extended corona like a thick atmosphere, but appears to be dominated by local major injection of hot (high speed) gas/dust plumes to high altitudes but only near active volcanic vents. Therefore, although the atmosphere is probably only locally thick, it still has a large extended neutral corona which might provide a sufficient source of impact ionization and photoionization to explain the plasma torus.

Neubauer [1980] presented an analytical model of the Alfvén standing wave current system which connects current through the ionosphere of Io. Southwood [1980] examined data from several Voyager instruments and examined the possible role of an intrinsic magnetic field for Io as a way to retain a robust enough ionosphere, which could provide enough conductivity for completing the lo-Jupiter current circuit. Several years after Voyager, 3D numerical studies of the plasma flow past lo were performed using electrodynamic [Wolf-Gladrow et al., 1987] and magnetohydrodynamic [Linker 1991] approaches.

The results of the measurements by the particle and field instruments on the Galileo Orbiter during the December 1995 flyby of Io provided new and important information with which realistic simulations for the plasma interaction can be tested. The J0 flyby occurred nearly in the equator plane of lo and perpendicular to a flow wake defined by the corotating plasma flow past Io. The spacecraft trajectory passed approximately $900 \mathrm{~km}$ down-stream of lo (in the sense of the plasma torus flow). Along that trajectory physical signatures of the wake were seen as a broad depression in the magnetic field [Kivelson et al., 1996], sharp peaks in the ion [Frank et al., 1996] and electron [Gurnett et al., 1996] densities, a slowing of the plasma in the core of the wake, a deep ion temperature decrease in the center of the wake, and a large (factor of 3 ) ion temperature rise in the flanks of the wake [Frank et al., 1996]. The magnetic field perturbation was broader spatially than the density peak, and showed a doublereversed structure, whereby the perturbation (as defined by the difference from the outer Jovian B-field value) was actually weaker right near the close-approach point than it was somewhat adjacent to the center of the wake.

With the goals to explain the general measured features from Galileo and to probe important properties of the interaction of the plasma torus with Io's neutral atmosphere and ionosphere, there have been recent efforts to improve and 


\section{PLASMA FLOW BY COMETS AND SATELLITES}

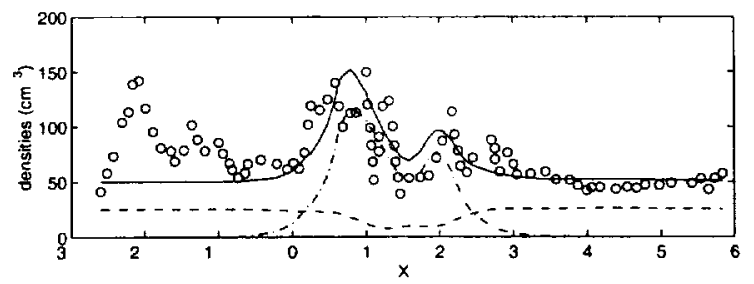

Figure 5. The calculated number densities and the observed electron density along the Galileo E4 flyby. The dashed line at the bottom gives the model density of the upstream plasma torus ions, and the dash-dot line gives the modeled density of the pickup ions. The electron density is constructed assuming the average charge state of the ambient torus ions is 2 , while that for the pickup $\mathrm{O}_{2}{ }^{+}$ions is one. The model electron density (solid line) with that inferred from the plasma wave measurements of Gurnett et al. [1998] (open circles).
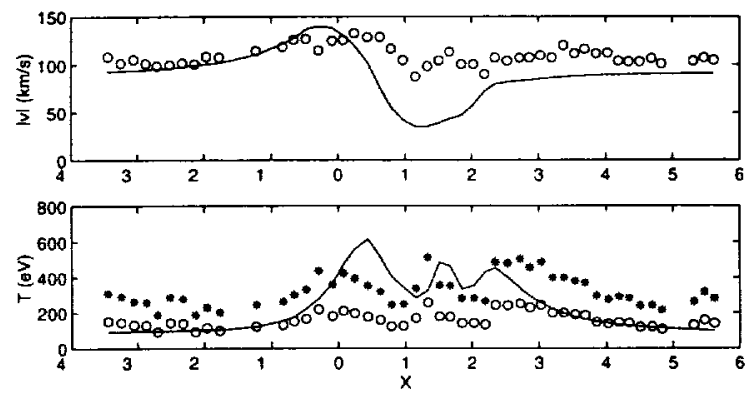

Figure 6. Speed and temperature profiles along the E4 flyby. The solid lines are the plasma speed (upper panel) and plasma temperature (lower panel) from the model results. The circles are the ion speed (upper panel and ion temperature (lower panel) data of Paterson et al. [1999]. Because the single-fluid plasma temperature of the MHD calculation is really the sum of the ion and electron temperature, which may be roughly comparable in this region of the torus, we show the values marked by the stars that are twice the measured ion temperature.

extend the pre-Galileo simulations both in terms of the MHD [Combi et al., 1998a; Linker et al., 1998; Kabin et al., 2001] and the electrodynamic [Saur et al. 1999] approaches. These two approaches are distinguished by the physical assumptions which they each do and do not (or in some cases, can and cannot) include.

The single-fluid MHD approach solves a system of mass, momentum, energy, and magnetic field conservation equations treating the plasma (ions and electrons) as a single fluid. Thus it can treat the deformation (draping) of magnetic field lines around the obstacle (the near lo region) and produce reasonably realistic flow geometry in three dimensions. The imposed outside (upstream) B-field can have any value and orientation with respect to the flow direction. However, MHD cannot, at least yet, include the effects of realistic conductivities (Hall and Pederson) or charge separation effects which are likely to be important very close to Io where the neutral densities are large and electric potentials can introduce non-symmetric flow around the body. They either include constant artificial conductivity [Linker et al., 1998] or assume perfect conductivity [Combi et al., 1998a], however comparisons of the two sets of published results do not indicate that this choice has any
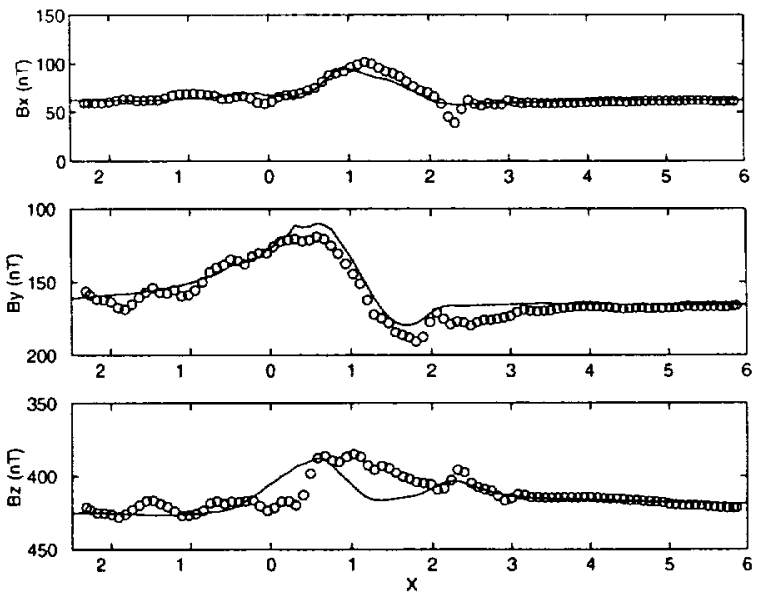

Figure 7. Magnetic field components along the E4 flyby. The solid lines are the results of the model. The circles are the data from the magnetometer measurements [Kivelson et al., 1997].

important consequence. Both MHD approaches address the finite conductivity at the inner boundary, either through solutions of Laplace's equation for simple conductivity models inside of Io in the case of Linker et al. [1998], or by fixing the B-field inside Io to be the time-averaged imposed Jovian field by Combi et al. [1998a]. The latter was shown previously by Linker et al. [1998] to be the major effect of actually solving Laplace's equation inside the inner boundary. The similarity of the results of the so-called fixed-boundary model of Combi et al. [1998a] and the so-called conducting model of Linker et al. [1998] seems to bear out the validity of the simpler approach, as well as the overall consistency of the two numerical codes despite their differences in numerical algorithms and computational grids.

Both sets of MHD calculations included mass-loading and charge exchange varying spatially as the power law distribution found by Schneider et al. [1991] for sodium. Linker et al. [1998] presented results for a conducting lo with no intrinsic magnetic field as well as for a magnetized lo, similar to the vacuum superposition field originally suggested by Kivelson et al. [1996]. Kabin et al. [2001] considered a somewhat more complicated aspherical neutral atmosphere based on a lower atmosphere with an exponential scale height distribution [Wong and Smyth, 2000] in addition to an extended power-law distribution from the corona, which resulted in some minor improvements. Both sets of MHD results produce a cold dense plasma wake for nominal levels of mass-loading and charge exchange. The non-magnetized models produce magnetic field perturbations that are similar to the Galileo measurements, but none are quite as deep or as broad, and none have the reversal of the pertubation (the double-peaked structure) in the center of the wake. The magnetized models of Linker et al. [1998] produced a broad and deep perturbation, but not the self-reversal at the center of the wake (the double-peak or bite-out). Figures 8 and 9 show a comparison of the magnetized model of Linker et al. [1998] and the non-magnetized models of Combi et al. [1998a] with the measurements. They do not enable a definitive statement to be made regarding an intrinsic field. A recent review of the later Io flybys still yields similarly ambiguous results [Kivelson et al., 2000] in this regard, although there is some hope of a conclusive answer from a successful polar flyby during the final Galileo extended mission. 
None of the standard MHD models have been able to reproduce the factor of three temperature rise in the flanks of the wake. This temperature rise is roughly coincident spatially with the broadened extent of the magnetic field perturbation. It was shown by Khurana et al. [1997b] that if the temperature increase were simply added in an ad hoc manner, it would account for all or most of the broadened extent of the magnetic field perturbation. In addition to including a more complicated neutral atmosphere distribution (as already mentioned), Kabin et al. [2001] also included deposition of pickup ions with their local corotation plus gyration energy in order to explain the broad magnetic field disturbance and the large temperatures in the flanks. The results generally verify the contention of Khurana et al., $1997 \mathrm{~b}$ ] but do not address the source of the extra energy. It is noteworthy to point out that the simulations of Linker et al. [1998] all assume that the ambient (upstream) plasma temperature is $200 \mathrm{eV}$, whereas the calculations of Combi et al. [1998a] adopted a plasma tempcrature $92 \mathrm{eV}$ from the measured ion results of Frank et al. [1996]. A careful comparison of the measured temperatures to the models of Linker et al. [1998] shows that they actually have the same small $10 \%$ increase of temperature in the flanks of the wake as the models of Combi et al. [1998a]. This increase is explained by the injection of nearly stationary pick-up ions into the supersonic plasma flow [Szegö et al., 2000]. The model temperature profile of Combi et al. [1998a] matches the Galileo data far from the wake $(92 \mathrm{eV})$ and in the center of the wake, but does not increase to $300 \mathrm{eV}$ in the flanks of the wake. The model temperature profile of Linker et al. [1998], on the other hand, is a factor of two too large away from the wake, cuts through the increase in the flanks, and then matches the cool observed temperatures in the center of the wake.

The electrodynamic approach Saur et al. [1999] starts from the assumptions that the magnetic field is everywhere uniform with the local Jupiter dipole value at lo, and that the flow is initially perpendicular to this field. The electric potential equation is solved in a series of planar cuts perpendicular to the magnetic field and through Io. The resulting flow is restricted from traveling along the magnetic field direction although it can be deflected around the obstacle within the plane from which it started. Hall and Pederson contributions to the conductivity are calculated using a neutral atmosphere having a scale height distribution similar to their previous work for Europa [Saur et al., 1998]. Their conductivity, however, goes to zero at a small altitude above the surface. They also include separate descriptions for ions and electrons. From the electric field described by the conductivity and charge separation, they calculated the deflected flow and the magnitude of the perturbation to the uniform B-field. Their source terms are self-consistent, rather than fixed as in the MHD models, however, at their present level of their model sophistication the magnetic field perturbation could not be fed-back into the plasma trajectory calculations self-consistently, so that they do not account for where the magnetic field is most strongly disturbed. Saur et al. [1999] performed many one-dimensional path-integral calculations along trajectories defined by the electric potential solution to obtain a full three-dimensional picture of ion and electron density, velocity, and temperature.
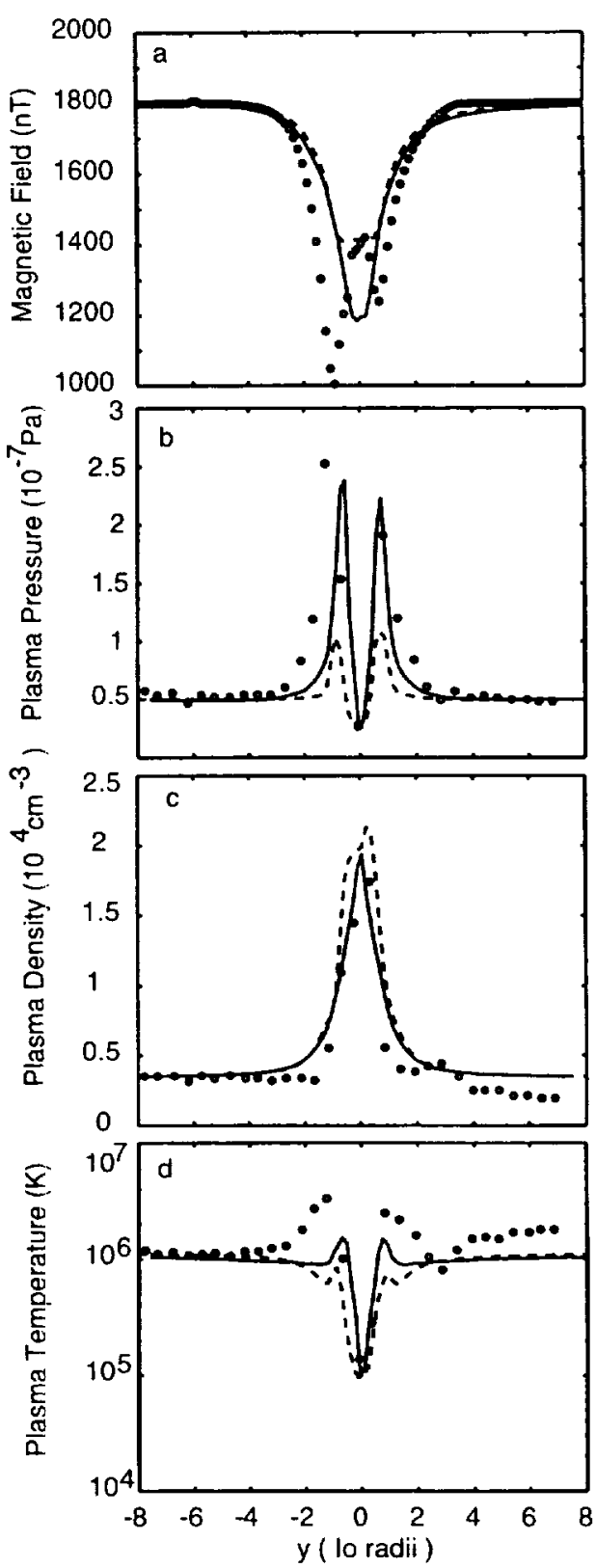

Figure 8. Comparison of the Mass-Loading Models with Galileo Particle and Field Measurements. The magnetic field measurements in (a) from Kivelson et al. [1996] and the plasma density (b), pressure (c), and temperature (d) from Frank et al [1996 obtained from the plasma ion instrument are shown with the Combi et al. [1998a] model values. The solid lines give the results for the model with mass-loading and reflective boundary conditions. The dashed lines give the results for the model with mass-loading and fixed boundary conditions.

Therefore, both the MHD and electrodynamic approaches have their strong and weak points. The high conductivity assumptions of the MHD calculations are more appropriate farther away from Io and the detailed conductivity 


\section{Magnetized Io}

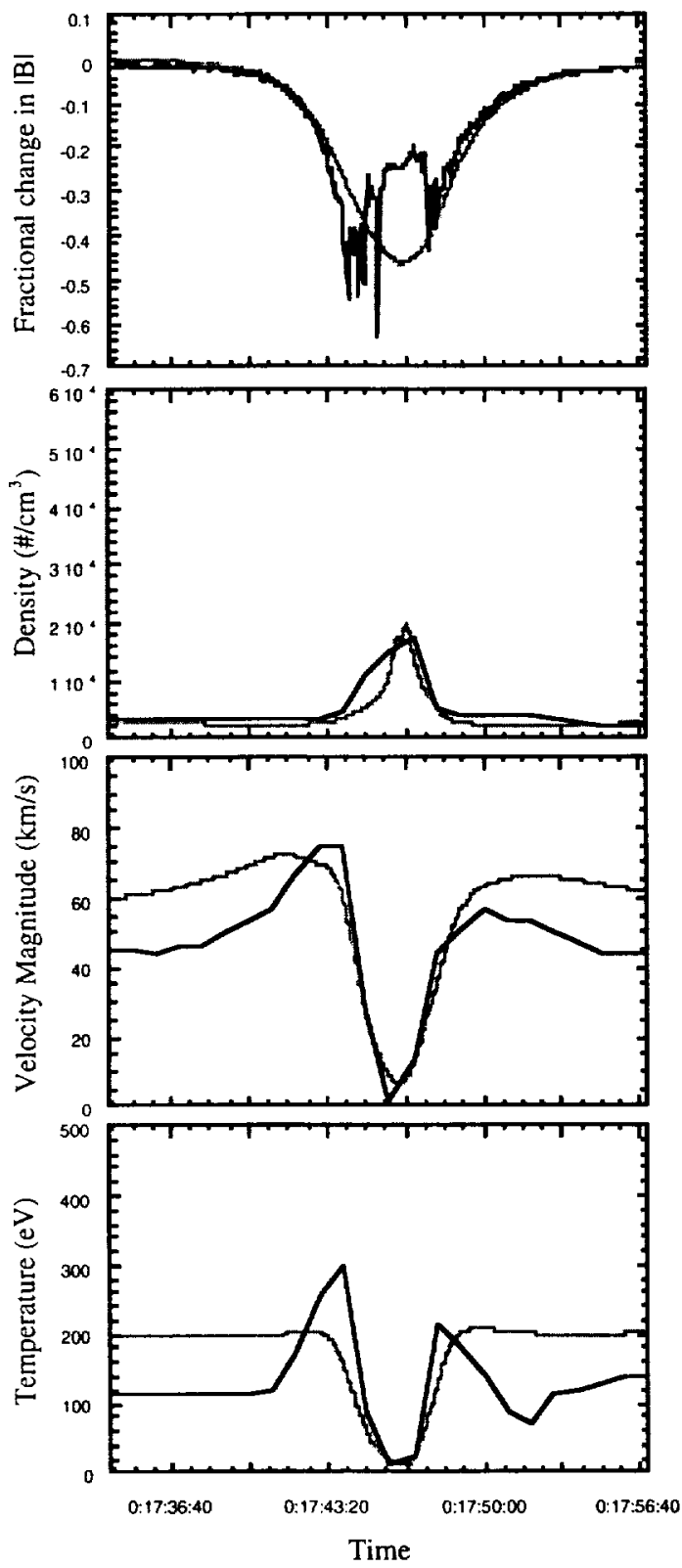

Figure 9. Comparison of simulated data traces from MHD computations of plasma flow past lo (gray curves) with the Galileo data (black curves). Results are from the magnetized model of Linker et al. [1998]. Data from the simulations were extracted along the Galileo trajectory for the Io flyby. (a) Fractional change in the magnitude of the magnetic field. Note the "double-valley" structure in the Galileo data. (b) Plasma density, (c) Velocity magnitude, and (d) Temperature.

calculations of the electrodynamic approach are better closer to Io's surface. Another important difference is that because the ion motion is restricted to the plane perpendicular to the average Jovian magnetic field, the flow in the electrodynamic approach is essentially incompressible (and inexpandable) and therefore cannot collapse in the wake behind lo as accomplished in MHD. Therefore, whereas the MHD calculations form a dense cold wake similar to the Galileo measurements, the approach of Saur et al. [1999] produces an empty wake.

In follow-up work published in his Ph.D. thesis, Saur [2000] shows that the combination of their assumed uniform B-field with their calculated perturbation field, can produce a double-peaked structure in their calculated magnetic field. However, this extra bite-out coincides with the location of the empty wake, and it is not clear that the extra bite-out would be present if their calculation produced the observed cold, dense wake as is observed and as the MHD calculations reproduce. Unfortunately, what was stated by Linker et al. [1998] remains true: no simulation to date has produced quantitatively and simultaneously all features of the Galileo measurements: the dense, cold plasma wake, the broad extent and the central bite-out in the $\mathrm{B}$-field across the wake, and the large temperature peaks in the flanks of the wake. Clearly simulation science has a long way to go before being able to address self-consistently the physically realistic complex nature of this coupled system. In the mean time, while acknowledging the strengths and weaknesses of both these and other approaches, much can still be gained by further studies using all of these approaches, which are complementary in many ways.

The electrodynamic approach could be generalized to include a realistic nonuniform magnetic field. This, among other things, would permit the flow to collapse behind the satellite and hopefully produce a realistic dense wake. The MHD approach could be generalized to include multiple species to account separately for newly deposited pickup ions and the background torus ions. Another possible refinement would be to include parallel and perpendicular temperatures (and pressures). It is quite clear that the newly deposited ions, either through mass-loading by new ions or by charge exchange, are introduced with a ring beam distribution. Both MHD and electrodynamic approaches need to better address the range of realistic conductivity around lo. In principle the MHD approaches treat the plasma to be perfectly conducting [Combi et al., 1998a]. The equations of Linker et al. [1998] showed an ohmic heating term, however it was not included in most of the simulations. They did include extra viscous terms to capture sharp fronts. The electrodynamic approach, on the other hand, assumes more realistic Hall and Pederson conductivities at low altitudes. This form of the conductivity goes to zero at finite altitude, rather than asymptotically approaching large conductivities dominated by ion-electron collisions at high altitudes [Song et al., 2001].

\subsection{Ideal MHD for Gyration Dominated Plasma at Io}

As discussed above, recent MHD models [Combi et al., 1998a; Linker et al., 1998] for the interaction of the Jovian plasma torus with the tenuous atmosphere of lo reproduced many aspects of the December 1995 Galileo Io fly-by measurements in the wake, such as the general flow geometry, the velocity perturbation, the density peak and the 
temperature trough [Frank et al., 1996; Gurnett et al., 1996b]. Only the intrinsic field model of Linker et al. [1998] produced a magnetic field signature approaching the breadth measured [Kivelson et al., [1996], and neither reproduced the measured temperature peaks in the flanks on either side of the wake [Frank et al., 1996], which exceeded the nearby $\sim 92 \mathrm{eV}$ torus values by a factor of 3 . Combi et al. [1998a] concluded that a process which adds energy in the flanks of the wake and/or upstream of the wake was required that was not included in the published MHD calculations. This was based in part on the suggestion by Khurana et al. [1997b] that if a temperature increase in the flanks were somehow added, a broader magnetic field disturbance would naturally result

There are some important issues regarding the structure of the neutral atmosphere which have been published since the previously published MHD models [Combi et al., 1998a; Linker et al., 1998], and so a new look is warranted. In addition, we have reconsidered one of the basic model parameters assumed before, the ratio of the specific heats, $\gamma$, which was chosen to be $5 / 3$. It is interesting to note that often in cometary and solar-wind pickup ion simulations, investigators have noted that in situations where the pickup ions remain in pickup ring distributions, it can be argued that there are really only two translational degrees of freedom because two are effectively tied together [Flammer et al., 1991; Ellison et al., 1999]. In this case, the appropriate value for $\gamma$ is 2 instead of $5 / 3$. It is quite clear from the Galileo magnetometer data that the ion-cyclotron wave signatures of pickup ions in the wake of lo are present. This is caused by the fact that neither collisions nor wave-particle instabilities are efficient enough to isotropize the distribution functions of particles during the time-scale of the plasma interaction near Io. In fact, the presence of a wake footprint for lo seen in Jovian aurorae [Clarke et al., 1998] more than half-way around Jupiter is an indication that particles may remain in a ring-beam distribution far downstream from Io. Although we might describe the background plasma with some thermal energy, it is clear that the nature of any magnetized plasma interaction is more gyration-like than isotropic in nature. Therefore, following the lead of other problems we explore here the effect of using a value for $\gamma$ of 2 instead of $5 / 3$.

For the first set of new model calculations presented in this paper the parameters remain the same as in the previous work of Combi et al. [1998a], except for taking $\gamma$ to be 2, and its effect on the Mach number which now also becomes 2.0 instead of 2.2. The purpose here is not to present a substantially new step, but rather to combine a few features from published work to explore possible improvements in an up-to-date MHD calculation. For this case we use the same $r$ spatial distribution for mass-loading and charge exchange as for both previous MHD models [Combi et al., 1998a; Linker et al., 1998], where $\mathrm{r}$ is the distance from the center of lo. The remaining model parameters are:

$\begin{array}{ll}\text { upstream plasma density } & =3500 \mathrm{~cm}^{-3} \\ \text { upstream plasma temperature } & =92 \mathrm{eV} \\ \text { upstream mean molecular mass } & =22 \mathrm{amu}\end{array}$

$\begin{array}{ll}\text { upstream magnetic field } & =1800 \mathrm{nT} \\ \text { corotation flow speed } & =56.8 \mathrm{~km} \mathrm{~s}^{-1} \\ \text { Alfvén Mach number } & =0.4 \\ \text { Mach number } & =2.0 \\ \text { ratio of specific heats }(\gamma) & =2\end{array}$

We use the fixed boundary conditions at Io which correspond to setting the plasma density, velocity and magnetic field in ghost cells at the inner boundary. A physically-consistent boundary condition either at Io (or at Jupiter for that matter) relates to the magnetic field perturbation via finite ionospheric conductivity. We must set values of plasma velocity and density and magnetic field at the boundary to achieve the desired result. As mentioned above, the fixing of an internal B-field at the average Jovian field level at Io's location produces the same results as assuming a constant finite conductivity inside the inner (lo) boundary in the explicit calculations of Linker [1991]. We again set the lower Io boundary at an altitude of $150 \mathrm{~km}$, the plasma density at $10500 \mathrm{~cm}^{-3}$, and the magnetic field at the time average value from Jupiter's dipole field at lo (1800 nT). In their recent paper Linker et al. [1998] performed a similar calculation with a somewhat more complicated layered conductivity model inside lo. However, the similarity between their conducting model and the fixed boundary model (not including the different assumed upstream torus plasma temperatures) indicates that the conditions at the lower boundary between the two MHD groups are in fact reasonably consistent with one another. Finally, as before the simulation volume for the new calculation is $900 \times 600 \mathrm{x}$ $600 \mathrm{R}_{\mathrm{fo}}$, and there are 9 levels of refinement and 92,000 cells with sizes ranging from $0.1 R_{10}$ near lo to $50 R_{10}$ far upstream and downstream.

The change of $\gamma$ to two seems to provide a natural mechanism to explain at least some of the previous modelmeasurement differences, namely the temperature peaks on either side of the flanks of wake. The previous fixedboundary model produced not only a density peak and temperature drop in the center of the wake and the general velocity field, but also a deeper magnetic field disturbance and a large increase in the temperature in the flanks of the wake, as shown by the thin solid lines in Figure 10. Also shown are the previous results [Combi et al., 1998a] indicated by the dashed lines. The mass-loading and chargeexchange rates are the same as the previous result. We make no attempt to improved the fit of the density peak by lowering the mass-loading but simply show what the effect is of changing $\gamma$.

\subsection{Mass-Loading by a More Realistic Neutral Atmosphere}

We also address here the spatial distribution of the mass loading and friction terms by incorporating a more realistic neutral atmosphere distribution. Essentially this combines the most realistic features of the extended neutral corona adoptcd in the MHD simulations [Combi et al., 1998a, 
Linker et al., 1998] with the scale height distribution of in lower atmosphere used in the electrodynamic simulation of [Saur et al., 1999] and some recent atmosphere models.

The $\mathrm{r}^{-3.5}$ spatial distribution for the ion mass-loading and charge exchange (friction) terms, which was adopted for the MHD simulations from the sodium eclipse measurements [Schneider et al., 1991], underestimates the expected rise in neutral density near lo. Models for the neutral atmosphere consist of a dense surface atmosphere near the surface temperature $(115-130 \mathrm{~K})$, and the corresponding scale height of only $\sim 12 \mathrm{~km}$ [Summers et al., 1996; Wong and Johnson, 1996, Wong: and Smyth, 2000]. In this region (which MHD models do not resolve anyway) there is not substantial ionization. At increasing altitudes, of importance for understanding the plasma interaction, the scale height increases to eventually to values on the order of $100-200 \mathrm{~km}$. At still larger distances a partially escaping corona forms with a power-law distribution like the sodium distribution as modeled by Smyth and Combi [1997]. To model the overall plasma interaction, a spatial profile was adopted which is consistent with these neutral atmosphere measurements and models. In particular, a scale height form for the near-surface atmosphere was chosen to be relevant for the altitudes that can be resolved by the computation grid and that merge into a power-law for higher altitudes.

In the region from 1.4 to $7 \mathrm{R}_{\mathrm{I}}$ a power-law distribution, varying as $\mathrm{r}^{-3.5}$ was found for the observations of the neutral sodium corona by Schneider et al. [1991]. However, because the bulk of the neutral corona does not consist of sodium but rather the major $\mathrm{SO}_{2}$ byproducts, $\mathrm{S}$ and $\mathrm{O}$, the earlier $\mathrm{r}^{-3.3}$ distribution may not be appropriate for the bulk constituents. The models of Smyth and Combi [1997], which reproduce the sodium corona measurements of Schneider et al. [1991], correspond to the non-thermal kinetic escape mechanism of Sieveka and Johnson [1984] with an most probable upward flux velocity of $0.5 \mathrm{~km} \mathrm{~s}^{-1}$. Sodium has an ionization potential of only $5.1 \mathrm{eV}$, and in typical plasma torus conditions has a lifetime against electron impact ionization that is a factor of 2 to 8 shorter than for $S$ and $O$, which have ionization potentials of 10.36 and $13.62 \mathrm{eV}$, respectively Accounting for the difference in the relative loss of upwardly moving $\mathrm{Na}$ atoms compared with the average of $\mathrm{S}$ and $\mathrm{O}$ implies that the bulk corona density (as opposed to just the trace amounts of neutral sodium) should decrease with increasing distance as $r^{-2.5}$ rather than the steeper $r^{-3.5}$ for $\mathrm{Na}$. Such a distribution is also consistent with some very recent Hubble Space Telescope measurements by Wolven et al. [2001, in press] of the UV emission by atomic $S$ and $O$.

Therefore, we have adopted a new form for the radial dependence of the mass-loading and charge-exchange rates to be $A \exp (-\mathrm{z} / \mathrm{H})+B \mathrm{r}^{-2.5}$, where $A$ and $B$ are constants adjusted during model-data comparison, $\mathrm{z}$ is the altitude, $\mathrm{r}$ is the distance from the center of $\mathrm{Io}$, and $\mathrm{H}$ is an effective scale height set to $114 \mathrm{~km}$ for the region in question. As before, the corona is cut-off at 7 Io radii from the center of lo. We adjust only one extra free parameter comparcd with previous MHD models, the relative contributions from the lower atmosphere and the extended corona, with the transition being around $2600 \mathrm{~km}$ from the center of 10 , and loosely corresponding to the atmospheric exobase. Ther inner Io boundary has been moved from $150 \mathrm{~km}$ above lo down to the surface, and three more levels of refinement were added making the size of the computational cells about $22 \mathrm{~km}$ near the surface and raising the total number of cells to about 150,000 .

In Figure 10 the Galileo PLS and MAG measurements are compared with the fixed-boundary model of Combi et al. [1998a], that model but with $\gamma=2$, and the two-component atmosphere model with $\gamma=2$. The two-component model reproduces the combination of magnetic field, plasma density and temperature better than either published MHD modeling attempts. The somewhat elevated temperatures in the flanks of the wake result from a combination of the use of $\gamma=2$ and the adjustment of the lower and upper atmosphere components. As before the velocity flow field does not change appreciably between the models, which appears essentially the same as shown by Combi et al. [1998a].

The total fresh ion mass-loading rate and the charge exchange rate, which contributes to the momentum and energy friction terms, is $1.2 \times 10^{28} \mathrm{~s}^{-1}$. The relative contributions from the inner atmosphere and outer corona are in the ratio of 15 to 1 . The new outer corona contribution is smaller than the previous model results. The velocity of ions is again similar to previous results, the variation of which matched the measurements by Frank et al. [1996]. The asymptotic velocity increase to corotation down the wake is similar to the values from the radio occultation measurements [Hinson et al., 1998] obtained in July 1997. These imply that the flow in the wake accelerates from near $30 \mathrm{~km} \mathrm{~s}^{-1}$ at around $2 R_{\mathrm{Io}}$ to nearly corotational speeds by only 5 to $7 \mathrm{R}_{\mathrm{I0}}$ down the wake. On the other hand, observations of the footprint of the wake of Io by Clarke et al. [1998], which is weakly present even half-way around the orbit of Io (hundreds of $\mathrm{R}_{\mathrm{lo}}$ ), have been interpreted as indicating a persistence of subcorotational flow far down the wake.

The reversal of the magnetic field disturbance at the center of the wake [Kivelson et al., 1996] is still not reproduced; the magnetic field disturbance in $B_{2}$ is still not broad enough; and the temperature peaks in the flanks are not extended spatially enough. However, with the modified neutral atmosphere distribution, the new model results do provide small temperature peaks and at least an inflection point and a flat distribution in $B_{z}$ at the right locations. There are a number of possible explanations, which new measurements and modeling studies might provide. The first would be an intrinsic magnetic field at Io. The 124 flyby of lo [Linker et al., 1999a] is inconclusive regarding an intrinsic field, and unfortunately the failure of the 125 flyby will not yield the anticipated conclusive measurements. It is possible that the recently announced flyby in the last extended Galileo mission phase may eliminate that as a viable explanation. The spatial characteristics of the mass-loading and charge exchange source rates may just be complicated. None of the MHD calculations include a self-consistent ionization source which is based on electron impact ionization of a given neutral atmosphere. More realistic simulations could also involve latitudinal and/or longitudinal variations, or irregular (volcanic) atmospheric distributions. Finally, the inclusion of other physical processes in simulations, such as those associated with the expected pressure anisotropy, multispecies effects, electron beams, currents, other nonequilibrium or kinetic effects, or other energy sources [Kabin 

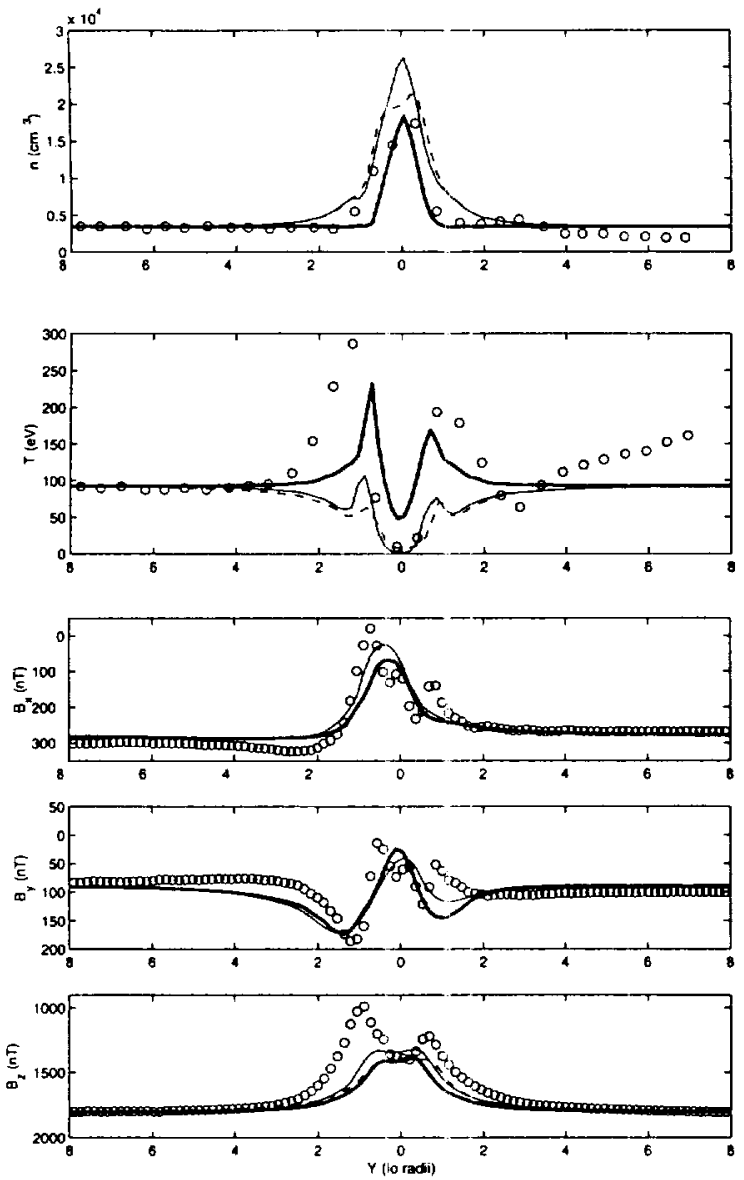

Figure 10. Comparison of lo MHD models results with Galileo measurements. Shown is a comparison of Galileo PLS [Frank et al., 1996] data and MAG [Kivelson et al., 1996] (the open circles) with originally published (dashed lines) results [Combi et al., 1998a], the same spatial model with $\gamma=2$ (thin solid lines), and a two-component atmosphere model with $\gamma=2$ (thick solid lines). The two top panels give the plasma density and temperature, and the bottom three give the magnetic field components.

et al., 2001; Khurana et al., 1997b] might be required to substantially improve the agreement between models and data.

\subsection{The Interaction of Titan with its Plasma Environs}

Titan possesses a dense local atmosphere [Kuiper, 1944; Lindal et al., 1983] which in the presence of an exterior magnetized plasma flow necessarily results in a strong and complicated interaction. Significant efforts have been invested into modeling Titan's upper atmosphere and ionosphere. These are discussed in chapters I.1., I.2 and I.3 of this monograph. Titan is also an interesting because it spends a large fraction of its orbit inside the Saturnian magnetosphere and a smaller part outside it, in the solar wind. This occurs when the solar wind flux is large and the
Saturnian bow shock is pushed closer to the planet. Therefore, the plasma environment around Titan can vary considerably. During the Voyager 1 encounter, Titan was clearly inside Saturn's magnetosphere.

Voyager 1 was the only spacecraft to visit the vicinity of Titan, having a closest approach on 12 November 1980 of 2.7 Titan radii from the center of Titan. These are the only magnetic field and plasma data available to the scientific community until Cassini has its first flyby of Titan in 2004. These measurements show a strong interaction between Titan and Saturn's magnetospheric plasma [Ness et al,. 1982; Hartle et al,. 1982]. The measurements and later analysis [Neubauer et al., 1984] suggest that Titan's magnetotail was produced by the draping of corotating magnetospheric field lines about Titan's ionosphere. The Saturnward aberration of the wake was ascribed to a deviation of the corotational flow by about $20^{\circ}$ from tangential. An asymmetry in the magnetic field strength was explained by the fact that the solar direction was at a large angle from the incident plasma flow, producing an inbound/outbound difference coincident with the dayside/nightside hemispheres. Neubauer et al. [1984] suggested that the difference is related to two different magnetic field-line draping regimes associated with light and heavy ions.

A number of comparisons were made between Titan and the classical induced (comet-like) magnetosphere of Venus [Kivelson and Russell, 1983; Veregin et al., 1984; Luhmann et al., 1991]. While the wake structure of Titan is similar to that of Venus, it must be remembered that the flow upstream of Titan is sub-Alfvénic in Saturn's magnetosphere whereas it is always super-Alfvénic at Venus. In the last few years, encouraged by computational and numerical advances and by the interest renewed by the upcoming Cassini missions a number of numerical simulation studies have been undertaken.

Modeling Titan's interaction with the Saturn magnetosphere, or the solar wind, is complicated by the fact that the validity of a fluid approach is questionable. Fluid descriptions (like ideal MHD) are only rigorously applicable in situations where the conductivity is high, the plasma parameter (number of charged particles in a Debye sphere) is large, the ion gyroradius is small compared to the typical length scale of the problem and characteristic speeds (sonic speed and Alfven speed) are much less than the speed of light. For Titan the only of these criteria that possibly becomes questionable is the gyroradius, which for a thermal $\mathrm{N}^{+}$ion is $2.25 \mathrm{R}_{\text {Titan }}$. For the much lighter $\mathrm{H}^{+}$, the gyroradius is only $0.16 \mathrm{R}_{\text {Titan. }}$. Far from Titan, this is not an issue, as long as computational cells are larger than gyroradius. However, close to Titan, both the plasma speed and temperature decrease (at $\sim 1.2 \mathbf{R}_{\text {Titan }}$ they are about a factor of four smaller than upstream values) while the magnetic field intensity is about a factor of two larger near the front stagnation point. This yields about an order of magntitude decrease in the gyroradius near the planet, keeping the conditions closer to the realm of ideal MHD. Luhmann [1996] further suggested that wave-particle interactions and plasma instabilities would also tend to decrease gyroradius.

Cravens et al. [1998] produced very useful three-species 2$D$ calculations of the plasma flow past Titan. The calculation 
successfully reproduced several main features of Titan's magnetospheric interaction, however, as in any 2-D model, it missed a number of important essentially 3-D effects. Ledvina and Cravens [1998] presented the first 3-D singlefluid calculations, where Titan was approximated as a region of high plasma density surrounded by an ion-producing shell. They used a nonuniform structured Cartesian grid with the small cell size of $0.1 \mathrm{R}_{\text {Titan }}$ at the surface of Titan and with upstream conditions imposed at $10 \mathbf{R}_{\text {Titan }}$ from the satellite. Their simulations produced a narrow wake and Alfvén wings, which are features detected by Voyager or expected from theoretical consideration. This and subsequents simulations found ion mass-loading rates on the order of $10^{25} \mathrm{~s}^{-1}$. Intercomparison of variations between models, in this regard, is complicated by differences in the models' inner boundary conditions, computational cell resolution, and number and classes of species.

Kabin et al. [1999] presented results for a 3-D single-fluid calculation making use of their adaptive grid numerical scheme which simultaneously included finer resolution near Titan as well as a much larger simulation domain. Their simulations included a well resolved central body and the better numerical method and computational grid was able to reveal more detailed structures in the magnetospheric interaction. Kabin et al. [2000] introduced a model with an internal magnetic field, which they suggested represented the induced field created by the ionosphere and which was able to better represent asymmetric aspects of the Voyager data. They also considered the effects of asymmetric mass-loading. Subequently, Kopp et al. [2001] argued that nonsymmetric mass-loading similar to that of Kabin et al. [2000] can be used to model the effects of the large ion gyroradius in a fluid description. The model of Kopp et al. [2001] provides an interesting connection between fluid and kinetic approaches. Another example of a combination of fluid and particle description is a work by Ledvina et al. [2000]. They have calculated the trajectories of the pickup ions in the prescribed magnetic field obtained from their earlier MHD simulation.

There have been two most recent simulations one in MHD and the other a hybrid simulation. The first is a multispecies MHD simulation by Nagy et al. [2001], which separately accounts for three generic species, light (e.g., $\mathbf{H}^{+}$, $\mathrm{H}_{2}{ }^{+}, \mathrm{H}_{3}{ }^{+}$), medium (e.g., $\mathrm{N}^{+}$and $\left.\mathrm{CH}_{3}{ }^{+}\right)$and heavy $\left(\mathrm{N}_{2}^{+}\right.$, and $\mathrm{HCNH}^{+}$). This permits the effects of exospheric mass loading, chemiscal reactions and ion-neutral collisions to be treated. It reproduced the global features (magnetic barrier, magnetotail and distributions of the major ionospheric species) and implied an escape flux of major ionospheric (heavy) species to be $6.5 \times 10^{24} \mathrm{~s}^{-1}$. The other is a full hybrid simulation, like those discussed by Brecht [chapter II.3], which has been performed by Brecht et al. [2000]. The hybrid simulation accounts for particle ions and fluid electrons in addition to computing the magnetic field. It includes a similar grouping of species classes. Although this approach is better justified for Titan, it is also unfortunately more computationally expensive, so as with the case of lo above, there are always trade-offs in comparing one simulation method to another.

The hybrid simulations of Brecht et al. [2000] quite naturally produced an asymmetic plasma flow around Titan, something that is not possible with the ideal MHD simulations. This is produced by the inclusion of Hall terms enabled by the explicit inclusion of finite gyroradius microphysics. However, the general scale of the larger disturbance created by Titan's ionosphere and mass-loading is actually quite similar to the MHD simulations.

\section{FINAL COMMENTS}

The title of the paper by Cloutier et al. [1978], which we have cited elsewhere in this review, is "A cometary ionosphere model for lo." This clearly portends the subsequent scientific and methodological connections between planetary satellites and comets in the space science literature. In fact the reference sections of papers in both fields show clearly that each field benefits from the contributions of observers, experimentalists, and theoreticians from the other. Here we have presented a review of the many common properties, features and modeling approaches of the tenuous atmospheres, ionospheres, and outer radiation, fields and particles environs of comets and outer planet satellites. Although much progress has been made in understanding global structures and local measurements, there is much work to do in terms of fundamental theoretical modeling of the combination of global morphologies with local microphysics. As computation power continues to advance during the near future it will be more and more possible to incorporate complicated detailed physical processes in our models. The challenge will be to stay at the forefront, constantly stretching computational capabilities, in order to improve our understanding of these fascinating subjects.

Acknowledgments. This work was supported by NASA grants NAG5-4318, NAG5-8942, and HPCC CAN NCCS5-146, and the NSF-NASA-AFOSR interagency grant NSF ATM-9318181.

\section{REFERENCES}

Allen, M., M. Delitsky, W. Huntress, Y. Yung, and W.-H. Ip, Evidence for methane and ammonia in the coma of comet P/Halley, Astron. Astrophys., 187, 502-512, 1987.

Austin, J. V., and D. B. Goldstein, Rarefied gas model of Io's sublimation-driven atmosphere, Icarus, 148, 370-383, 2000.

Bigg, E. K., Influence of the satellite Io on Jupiter's decametric emission, Nature, 203, 1008-1010, 1964.

Bird, G. A., Molecular Gas Dynamics, Clarendon Press, Oxford, 1976.

Bird, G. A., Molecular Gas Dynamics and the Direct Simulation of Gas Flows, Clarendon Press, Oxford, 1994.

Biver, N., et al., Long-term evolution of the outgassing of comet Hale-Bopp from radio observations, Earth, Moon and Planets, $78,5-11,1997$.

Bockelée-Morvan, D., and J. Crovisier, The role of water in the thermal balance of the coma, in Diversity and Similarity of Comets, pp. 235-240, 1987.

Brecht, S. H, J. G. Luhmann, and D. J. Larson, Simulation of the Saturnian magnetospheric interaction with Titan, J. Geophys. Res., 105, 13,119-13,130, 2000.

Brooke, T. Y., A T. Tokunaga, H. A. Weaver, J. Crovisier, D. Bockelee-Morvan, and D. Crisp, Detection of acetylene in the infrared spectrum of comet Hyakutake, Nature, 383, 606-608, 1996. 
Brown, M. E., and R. E. Hill, Discovery of an extended sodium atmosphere around Europa, Nature, 380, 229-231, 1996.

Clarke, J. T., et al., Hubble Space Telescope imaging of Jupiter's UV aurora during the Galileo orbiter nission, J. Geophys. Res., 103(E9), 20,217-20,236, 1998.

Cloutier, P. A, R. E. Daniell, A J. Dessler, and T. W. Hill, A cometary ionosphere model for Io, Astrophys. Space Sci., 55, 93-112, 1978.

Coates, A J., A. D. Johnstone, B. Wilken, and F. Neubauer, Velocity space diffusion and nongyrotropy of pickup water group ions at comet Grigg-Skjellenup, J. Geophys. Res., 98, 20,995-21,002, 1993.

Cochran, A. L., Nonequilibrium chemical analysis of the coma of comet Stephan-Oterma, Icarus, 62, 82-96, 1985.

Colom, P., E. G' erard, J. Crovisier, D. Bockel' e-Morvan, N. Biver, and $\mathrm{H}$. Rauer, Observations of the $\mathrm{OH}$ radical in comet $\mathrm{c} / 1995$ o1 (Hale-Bopp) with the Nancay Radio Telescope, Earth, Moon and Planets, 78, 37-43, 1997.

Combi, M. R., Sources of cometary radicals and their jets - gases or grains, Icarus, 71, 178-191, 1987

Combi, M. R., The outflow speed of the coma of Halley's comet, Icarus, 81, 41-50, 1989.

Combi, M. R., Time-dependent gas kinetics in tenuous planetary atmospheres: The cometary coma, Jcarus, 123, 207-226, 1996.

Combi, M. R. and A. H. Delsermme, Neutral cometary atmospheres. I - An average random walk model for photodissociation in comets, Astrophys. J., 237, 633-640, 1980

Combi, M. R., and P. D. Feldman, IUE observations of H Lymanalpha in Comet P/Giacobini-Zinner, Icarus, 97, 260-268, 1992.

Combi, M. R., and U. Fink, A critical study of molecular photodissociation and CHON grain sources for cometary $\mathrm{C}$, Astrophys. J., 484, 879, 1997.

Combi, M. R, and W. H. Smyth, Monte Carlo particle-trajectory models for neutral cometary gases.I -Models and equations, Astrophys. J., 327, 1026-1043, 1988a.

Combi, M. R, and W. H. Smyth, Monte Carlo particle trajectory models for neutral cometary gases. Il. The spatial morphology of the Lyman-alpha coma, Astrophvs. J., 327, 1044-1059, $1988 \mathrm{~b}$.

Combi, M. R., B. J. Bos, and W. H. Smyth, The $\mathrm{OH}$ distribution in cometary atmospheres - A collisional Monte Carlo model for heavy species, Astrophys. J., 408, 668-677, 1993.

Combi, M. R., K. Kabin, T. Gombosi, D. De Zeeuw, and K. Powell, Io's plasma environment during the Galileo flyby: Global three-dimensional MHD modeling with adaptive mesh refinement, J. Geophys. Res., 103(A5i, 9071-9081, 1998.

Combi, M. R., K. Kabin, T. Gombosi, D. De Zeeuw, and K. Powell, Dust-gas interrelations in comets: observations and theory, Earth, Moon and Planets, 79, 275-306, 1999

Cravens, T. E., C. J. Lindgren, and S. A Ledvina, A twodimensional multifluid MHD model of Titan's plasma environment, Planet. Space Sci. 46, 1193-1205, 1998.

Crifo, J. F., and A. V. Rodionov, Modelling the circumnuclear coma of comets: objectives, methods and current results, Planet. Space Sci., 47, 797-826, 1999.

Crifo, J. F., A. L. Itkin, and A. V. Rodionov, The near-nucleus coma formed by interacting dusty gas jets effusing from a cometary nucleus: I, Icarus, 116, 77-112, 1995

Crifo, J. F., A. V. Rodionov, and D. Bockelée-Morvan, The dependence of the circumnuclear coma structure on the properties of the nucleus, lcarus, 138, 85-106, 1999.

Crovisier, J., Infrared Observations of Volatile Molecules In Comet Hale-Bopp, Earth Moon and Planets, 79, 125-143, 1999.
Ellison, D. C., F. C. Jones, and M. G. Baring, Direct acceleration of pickup ions at the solar wind termination shock: The production of anomalous cosmic rays, Astrophys. J., 512, 403-416, 1999

Festou, M. C., The density distribution of neutral compounds in cometary atmospheres. I -Models and equations, Astron. Astrophys., 95, 69-79, 1981.

Flammer, K. R., D. A. Mendis, E. C. Whipple, and T. G. Northrop, A self-consistent model for the particles and fields upstream of an outgassing comet. 1 - Gyrotropic and isotropic ion distributions, J. Geophys. Res., 96, 15907-15916, 1991.

Frank, L. A., W. R. Paterson, K. L. Ackerson, V. M. Vasyliunas, F. V. Coroniti, and S. J. Bolton, Plasma observations at lo with the Galileo spececraft, Science, 274, 394-395, 1996.

Giguere, P. T., and W. F. Huebner, A model of comet comae. I Gas-phase chemistry in one dimension, Astrophys. J., 223, 638-654, 1978

Goertz, C. K., Io's interaction with the plasma torus, J. Geophys. Res. 85, 2949-2956, 1980.

Goldreich, P., and D. Lynden-Bell, lo, a Jovian unipolar inductor, Astrophys. J., 156, 59-78, 1969.

Gombosi, T. I., A. F. Nagy, and T. E. Cravens, Dust and neutral gas modeling of the inner atmospheres of comets, Rev. Geophys. 24(3), 667-700, 1986

Gurnett, D. A., and W. S. Kurth, Radio emissions from the outer heliosphere, Space Sci. Rev., 78, 53-66, 1996

Gurnett, D. A, W. S. Kurth, A. Roux, S. J. Bolton, and C. F. Kennel, Galileo plasma wave observations near the Io plasma torus near Io, Science, 274, 391-392, 1996.

Gurnet, D. A., W. S. Kurth, A. Roux, S. J. Bolton, E. A. Thomsen, and J. B. Groene, Galileo plasma wave observations near Europa, Geophys. Res. Lett., 25, 237-240, 1998.

Häberl, R., M. R. Combi, T. I. Gombosi, D. L. De Zeeuw, and K. G. Powell, Quantitative analysis of $\mathrm{H}_{2} \mathrm{O}^{+}$coma images using a multiscale $\mathrm{MHD}$ model with detailed ion chemistry, Icarus. $130,373-386,1997$

Hall, D. T., D. F. Strobel, P. D. Feldman, M. A. McGrath, and H. A. Weaver, Detection of an oxygen atmosphere on Jupiter's moon Europa, Nature, 373, 677-680, 1995.

Harris, W. M., M. R. Combi, R. K. Honeycutt, and B. E. A. Mueller, Evidence for interacting gas flows and an extended volatile source distribution in the coma of comet C/1996 B2 (Hyakutake), Science, 277, 676-681, 1997.

Hartle, R.E., E.C. Sittler, KW. Ogilvie, J.D. Scudder, A.J. Lazarus, and S.K. Atreya, Titan's ion exosphere oberved from Voyager 1, J. Geophys. Res., 87, 1383-1394, 1982.

Haser, L., Distribution d'intensite dans la tete d'une comete, Bull. Acad. Roy. de Belgique, Classe de Sci., 43(5), 740-750, 1957.

Haser, L. Calcul de distribution d'intensite relatice dans une tete cometaire, Mem. Soc. Roy. Sci. Liege, 12(5), 233-24I, 1966

Hill, T. W., A. J. Dessler, and C. K. Goertz, Magnetospheric models, in Physics of the Jovian Magnetosphere, edited by A. J. Dessler, pp. 353-395, Cambridge University Press, New York, 1983

Hinson, D. P., A J. Kliore, F. M. Flasar, J. D. Twicken, P. J. Schinder, and R. G. Herra, Galileo radio occultation measurements of Io's ionosphere and plasma wake, $J$. Geophys. Res., 103(A12), 29343-29357, 1998.

Hodges, R. R., Monte Carlo simulation of nonadiabatic expansion in cometary atmospheres -Halley, Icarus, 83, $410-433,1990$.

Huebner, W. F., and J. J. Keady, Energy balance and photochemical processes in the inner coma, in Cometary Expioration. I, edited by T. I. Gombosi, pp. 165-183, Central Research Institute for Physics, Budapest, Hungary, 1983. 
Ip, W., Photochemical heating of cometary comae. III - The radial variation of the expansion velocity of $\mathrm{CN}$ shells in comet Halley, Astrophys. J., 346, 475-480, 1989.

Ip, W., Europa's oxygen exosphere and its magnetospheric interaction, Icarus, 120, 317-325, 1996.

Kabin, K., M. R. Combi, T. I. Gombosi, A. F. Nagy, D. L. DeZeeuw, and K. G. Powell, On Europa's magnetospheric interaction: An MHD simulation of the E4 flyby, J. Geophys. Res., 104(A9), $19,983-19,992,1999$.

Kabin, K, M. R. Combi, T. I. Gombosi, D. L. DeZeeuw, K. C. Hansen, and K. G. Powell, Io's magnetospheric interaction: an MHD model with day-night asymmetry, Planet. Space Sci., 49, $337-344,2001$.

Kabin, K, T. I. Gombosi, D. L. DeZeeuw, K. G. Powell, and P.L. Israelevich, Interaction of the Saturnian magneosphere with Titan: Results of a 3D MHD simulation, J. Geophys. Res., 104, 2451-2458, 1999.

Kabin, K., P. L. Israelevich, A. I. Ershkovich, F. M. Neubauer, T. I. Gombosi, D. L. de Zeeuw, K. G. Powell, Titan's magnetic wake: Atmospheric or magnetospheric interaction, J. Geophys. Res., $105,10,761-10,770,2000$.

Keller, $H$., et al., First Halley multicolour imaging results from Giotto, Nature, 321, 320-326, 1986.

Keller, H. U., and R. R. Meier, A cometary hydrogen model for arbitrary observational geometry, Astron. Astrophys., 52, 273-281, 1976.

Khurana, K. K., M. G. Kivelson, and C. T. Russell, Interaction of Io with its torus: Does it have an internal magnetic field?, Geophys. Res. Lett., 24, 2391-2394, 1997.

Khurana, K. K., M. G. Kivelson, D. J. Stevenson, G. Schubert, C. T. Russell, R J. Walker, and C. Polanskey, Induced magnetic fields as evidence for subsurface oceans in Europa and Callisto, Nature, 395, 777-780, 1998.

Kitamura, Y., Axisymmetric dusty gas jet in the inner coma of a comet, Icarus, 66, 241-257, 1986.

Kitamura, Y., O. Ashihara, and T. Yamamoto, A model for the hydrogen coma of a comet, Icarus, 61, 278-295, 1985.

Kivelson, M. G., Ambiguities and expectations: Io's magnetic signature and magnetospheric interactions, in AAS/Division of Planetary Sciences Meeting, vol. 32, p. 2805, 2000.

Kivelson, M. G., K K Khurana, R. J. Walker, J. Warnecke, C. T. Russell, J. A. Linker, D. J. Southwood, and C. Polanskey, Io's interaction with the plasma torus: Galileo magnetometer report, Science, 274, 396-398, 1996.

Kivelson, M. G., K. Khurana, S. Joy, C. Russell, D. Southwood, R. J. Walker, and C. Polanskey, Europa's magnetic signature: Report from Galileo's pass on 19 December 1996, Science, 276, 1239-1241, 1997.

Kivelson, M.G. and C.T. Russell, The interaction of flowing plasmas with planetary atmospheres: A Titan-Venus comparison, J. Geophys. Res., 88, 49-57, 1983.

Kliore, A. J., D. Hinson, F. Flasar, A. Nagy, and T. Cravens, The ionosphere of Europa from Galileo radio occultations, Science, 277, 355-358, 1997.

Kömle, N. I., and W. H. Ip, Anisotropic non-stationary gas flow dynamics in the coma of comet $\mathrm{P} /$ Halley, Astron. Astrophys., $187,405,1987$.

Kopp, A. and W.-H. Ip, Asymmetric mass loading effect at Titan's ionosphere, J. Geophys. Res., 106, 8323-8332, 2001.

Körösmezey, A., and T. I. Gombosi, A time-dependent dusty gas dynamic model of axisymmetric cometary jets, Icarus, 84, $118-153,1990$.

Kuiper, G. P., A satellite with an atmosphere, Astrophys. J., 100, 378-383, 1944.

Ledvina, S. and T.E. Cravens, A three-dimensional MHD model of plasma flow around Titan, Planet. Space Sci. 46, 1175-1191, 1998.
Ledvina, S. A., T. E. Cravens, A Salman, K. Kecskemety, Ion Trajectories in Saturn's Magnetosphere Near Titan, Advances in Space Research, 26, 1691-1695, 2000.

Lellouch, E., Urey Prize Lecture. Io's Atmosphere: Not yet understood, Icarus, 124, 1-21, 1996.

Lindal G. F., G. E. Wood, H. B. Hotz, D. N. Sweetnam, V. R. Eshleman, and G. L. Tyler, The atmosphere of Titan: An analysis of the Voyager 1 radio occultation measurements, Icarus, 53, 348-363, 1983.

Linker, J., M. McGrath, M. Kivelson, R. Walker, and K. Khurana, MHD modeling of Io's interaction with the plasma torus: Prediction for the upcoming Galileo flybys, in $A A S / D i v i s i o n$ of Planetary Sciences Meeting, vol, 31, p. 7809, 1999.

Linker, J. A, M. G. Kivelson, and R. J. Walker, A threedimensional MHD simulation of plasma flow past $I 0, J$. Geophys. Res., 96, 21,037-21,053, 1991.

Linker, J. A., K. K. Khurana, M. G. Kivelson, and R. J. Walker, MHD simulation of Io's interaction with the plasma torus, $J$. Geophys. Res., 103(E9), 19,867-19,877, 1998.

Luhmann J. G., C.T. Russell, K. Schwinginschuh, and Y. Yeroshenko, A comparison of induced magnetotails of planetary bodies: Venus, Mars and Titan, J. Geophys. Res, 96, $11,199-11,208,1991$.

Luhmann, J. G., Titan's ion exosphere wake: A natural mass spectrometer?, J. Geophys. Res. 101, 29,387-29,393, 1996.

Liu, Y., A. F. Nagy, K. Kabin, M. R. Combi, D. L. Dezeeuw, T. I. Gombosi, and K. G. Powell, Two-species, 3D, MHD simulation of Europa's interaction with Jupiter's magnetosphere, Geophys. Res. Lett., 27, 1791-1794, 2000.

Marconi, M. L, and D. A. Mendis, The atmosphere of a dirtyclathrate cometary nucleus - a two-phase, multifluid model, Astrophys. J., 273, 381-396, 1983.

Marconi, M. L., L. Dagum, and W. H. Smyth, Hybrid fluid/kinetic approach to planetary atmospheres: an example of an intermediate-mass body, Astrophys. J., 469, 393-401, 1996.

Miller, R. H., and M. R. Combi, A Coulomb collision algorithm for weighted particle simulations, Geophys. Res. Lett., 21 , 1735-1738, 1994.

Miller, R. H., C. E. Rasmussen, M. R. Combi, T. I. Gombosi, and D. Winske, Ponderomotive acceleration in the auroral region: A kinetic simulation, J. Geophys. Res., 100, 23901-23916, 1995.

Mitchell, G. F., S. S. Prasad, and W. T. Huntress, Chemical model calculations of $\mathrm{C}, \mathrm{C}, \mathrm{CH}, \mathrm{CN}, \mathrm{OH}$, and $\mathrm{NH}$ abundances in cometary comae, Astrophys. J., 244, 1087-1093, 1981.

Motschmann, U., and K. Glassmeier, Nongyrotropic distribution of pick-up ions at comet P/Grigg-Skjellerup: a possible source of wave activity, J. Geophys. Res., 98, 20,977-20,983, 1993.

Nagy, A. F., Y. Liu, K. C. Hansen, K Kabin, T. I Gombosi, M. R. Combi, and D. L. DeZeeuw, The interaction between Saturn's magnetosphere and Titan's ionosphere, J. Geophys. Res., 106, 6151-6160, 2001.

Ness, N.F., M.H. Acuna, KW. Behannon, and F.M. Neubauer, The induced magnetosphere of Titan, J. Geophys. Res. 87, 1269 $1282,1982$.

Neubauer, F. M., Nonlinear standing Alfvén wave current system at Io - Theory, J. Geophys. Res., 85, 1171-1178, 1980.

Neubauer, F. M., The sub-Alfvénic interaction of the Galilean satellites with the Jovian magnetosphere, J. Geophys. Res., 103, 19,834-19,866, 1998.

Neubauer, F. M., Alfvén wings and electronmagnetic induction in the interiors: Europa and Callisto, J. Geophys. Res., 104, 28671-28684, 1999.

Neubauer, F. M., D.A. Gurnett, J.D. Scudder, and R. E. Hartle, Titan's magnetospheric interaction, in Saturn, edited by $T$. Gehrels and M.S. Matthews, pp. 760-787, Univ. of Ariz. Press, Tucson, 1984. 
Neugebauer, M., R. Goldstein, B. E. Goldstein, S. A. Fuselier, H. Balsiger, and W. Ip, Densities and abundances of hot cometary ions in the coma of P/Halley, Astrophys. J., 372, 291-300, 1991.

Paranicas, C., R. W. McEntire, A F. Cheng, A. Lagg, and D. J. Williams, Energetic charged particles near Europa, J. Geophys. Res., 105, 16,005-16,016, 2000.

Paterson, W. R., L. A. Frank, and K. L. Ackerson, Galileo plasma observations at Europa: lon energy spectra and moments, $J$. Geophys. Res., 104, 22,779-22,792, 1999.

Pospieszalska, M. K, and R. E. Johnson, Plasma heating of Io's atmosphere, Geophys. Res. Lett., 19, 949-952, 1992.

Pospieszalska, M. K. and R. E. Johnson, Monte Carlo calculations of plasma ion-induced sputtering of an atmosphere: SO ejected from lo, J. Geophys. Res., 101, 7565-7574, 1996.

Rauer, H., F. Roesler, F. Scherb, H. U. Schmidt, and R. Wegmann, Ion emission line profiles in cometary plasma tails., Astron. Astrophys., 325, 839-846, 1997.

Russell, C. T., and D. E. Huddleston. The Unipolar Inductor Myth: Mass Addition or Motional Electric Field as the Source of Field-aligned Currents at lo, Advances in Space Research, 26, 1665-1670, 2000.

Saur, J., Plasma interaction of Jo and Europa with the Jovian Magnetosphere, Ph.D. thesis, Universität zu Köln, 2000.

Saur, J., D. Strobel, and F. Neubauer, Interaction of the Jovian magnetosphere with Europa: Constraints on the neutral atmosphere, J. Geophys. Res., 103, 19,947-19,962, 1998.

Saur, J., F. M. Neubauer, D. F. Strobel, and M. E. Summers, Threedimensional plasma simulation of Io's interaction with the Io plasma torus: Asymmetric plasma flow, J. Geophys. Res., 104, $25,105-25,126,1999$

Schneider, N. M., D. M. Hunten, W. K. Wells, A. B. Schultz, and U. Fink, The structure of Io's corona, Astrophys. J., 368, $298-315,1991$.

Shematovich, V. I., D. V. Bisikalo, and J. C. Gerard, A kinetic model of the formation of the hot oxygen geocorona. 1: Quiet geomagnetic conditions, $J$. Geophys. Res., 99, 23,217-23,229, 1994.

Sieveka, E. M., and R. E. Johnson, Ejection of atoms and molecules from lo by plasma-ion impact, Astrophys. J., 287, $418-426,1984$

Skorov, Y. V. and H. Rickman, Simulation of gas flow in a cometary Knudsen layer, Planet. Space Sci., 46, 975-996, 1998.

Smyth, W. H., and M. R. Combi, Io's sodium exosphere and spatially extended cloud: A consistent flux speed distribution, Icarus, 126, 58-77, 1997.

Song, P., T. I. Gombosi, and A. J. Ridley, Three-fluid Ohm's law, J. Geophys. Res., 106, 8149-8156, 2001.

Southwood, D. J., M. G. Kivelson, R. J. Walker, and J. A. Slavin, Io and its plasma environment, J. Geophys. Res., 85, 5959-5968, 1980.

Summers, M., and D. Strobel, Photochemistry and vertical transport in Io's atmosphere and ionosphere, Icarus, 20, 290-316, 1996.

Szegö, K., et al., Physics of mass loaded plasmas, Space Science Reviews, 94, 429-671, 2000.

Veregin, M. I., K. I. Gringauz, and N. F. Ness, Comparison of induced magnetospheres at Venus and Titan, J. Geophys. Res., $89,5461-5470,1984$.

Wallace, L. V., and Miller, F. D. , Isophote configurations for model comets, Astron. J., 63, 21 3-219, 1958.

Wilson, G. R., J. L. Honwitz, and J. Lin, A semikinetic model for early stage plasmasphere refilling I -Effects of Coulomb collisions, J. Geophys. Res., 97, 1109-1119, 1992.
Wolf-Gladrow, D. A., F. M. Neubauer, and M. Lussem, Io's interaction with the plasma torus: A self-consistent model, $J$. Geophys. Res., 92, 9949-9961, 1987.

Wong, M., and R. Johnson, A three-dimensional azimuthally symmetric model atmosphere for lo. 1. Photochemistry and the accumulation of a nightside atmosphere, J. Geophys. Res., 101, 23,343-23,254, 1996.

Wong, M. C., and W. H. Smyth, Model calculations for 10's atmosphere at eastern and western elongations, Icarus, 146, 60-74, 2000.

Xie, X., and M. J. Mumma, Monte Carlo simulation of cometary atmospheres: Application to comet P/Halley at the time of the Giotto spacecraft encounter. I. Isotropic model, Astrophys. J., 464, 442-456, 1996a.

Xie, X., and M. J. Mumma, Monte Carlo simulation of cometary atmospheres: Application to comet $\mathrm{P} / \mathrm{Halley}$ at the time of the Giotto spacecraft encounter. II. Axisymmetric model, Astrophys. J., 464, 457-475, $1996 \mathrm{~b}$.

Zimmer, C., K. K. Khurana, and M. G. Kivelson, Subsurface Oceans on Europa and Callisto: Constraints from Galileo Magnetometer Observations, Icarus, 147, 329-347, 2000.

Michael R. Combi and Tamas I. Gombosi: Department of Atmospheric, Ocean and Space Sciences, University of Michigan, 2455 Hayward Street, Ann Arbor, Michigan.

Konstantin Kabin: Department of Physics, University of Alberti, Edmonton, Alberta, Canada 
